\title{
Beam-beam amplitude detuning with forced oscillations
}

\author{
R. Tomás* and X. Buffat \\ CERN, CH-1211 Geneva, Switzerland
}

S. White

ESRF, 38000 Grenoble, France

\author{
J. Barranco, P. Gonçalves Jorge, and T. Pieloni \\ EPFL, CH-1015 Lausanne, Switzerland
}

(Received 12 July 2017; published 9 October 2017)

\begin{abstract}
Recently, relations were established between the coefficients of free and forced amplitude detuning polynomial expansions. The forced oscillations were considered only in a single plane. In this paper we extend and generalize previous results by developing analytical equations that transform the free amplitude detuning function into the amplitude detuning involving forced oscillations in both transverse planes. These are used to obtain closed approximated formulas for the beam-beam amplitude detuning with forced oscillations. Formulas are compared to single and multiparticle simulations.
\end{abstract}

DOI: 10.1103/PhysRevAccelBeams.20.101002

\section{INTRODUCTION}

Forced oscillations play a very important role in modern accelerators. They may be induced by magnets with imperfect power supplies or by radio frequency noise in deflecting devices, like crab cavities [1], possibly leading to instabilities. Forced oscillations have been purposely used in accelerators to avoid spin resonances with AC dipoles [2]. Since then, AC dipoles have become key instruments for the measurement and control of linear and nonlinear dynamics in hadron colliders [3-15]. Forced oscillations are also exploited in the study of beam-beam effects [16-18] and impedances [19]. Beam-beam effects in HL-LHC will possibly induce $\beta$-beating above the tolerances from machine protection [20] and forced oscillations with $\mathrm{AC}$ dipoles are promising instruments to measure the beam-beam $\beta$-beating. Experimental studies are ongoing at the LHC [21,22]. These would allow verifying and optimizing beam-beam correction schemes [23-27]. For all the above reasons it is important to gain understanding in the beam dynamics of forced oscillations.

In $[5,7,12]$ it is studied how the dynamics of forced oscillations differs from free oscillations. In particular, [12] shows that amplitude detuning coefficients change between the two types of motion depending on the multipolar order responsible of the amplitude detuning.

*rogelio.tomas@cern.ch

Published by the American Physical Society under the terms of the Creative Commons Attribution 4.0 International license. Further distribution of this work must maintain attribution to the author(s) and the published article's title, journal citation, and DOI.
Section II extends the result in [12] with analytical equations that transform the free amplitude detuning, $Q_{x, y}\left(J_{x}, J_{y}\right)$, into the amplitude detuning involving forced oscillations, $Q_{x, y}\left(J_{x}, J_{y}, A_{x}, A_{y}\right)$. The free and forced actions are represented by $J_{x, y}$ and $A_{x, y}$, respectively. Note that, abusing notation, we use the same $Q$ function for the free and forced cases as $Q_{x, y}\left(J_{x}, J_{y}\right)=Q_{x, y}\left(J_{x}, J_{y}, 0,0\right)$. Section III applies the result in Sec. II to the beam-beam amplitude detuning and compares analytical formulas to single and multiparticle simulations.

\section{RELATING FREE AND FORCED AMPLITUDE DETUNING FORMULAS}

Similarly as in [12] the average Hamiltonian can be expressed as

$$
\langle H\rangle=\oint \mathrm{d} s \sum_{j, k=0}^{\infty} h_{j k}\left\langle x^{2 j}\right\rangle\left\langle y^{2 k}\right\rangle,
$$

where $x$ and $y$ are the horizontal and vertical coordinates, the averaging denoted by \langle\rangle takes place over all phase variables. It is important to note that we have assumed that $x$ and $y$ coordinates are fully independent. The horizontal amplitude detuning is given by

$$
\Delta Q_{x}=\frac{1}{2 \pi} \frac{\partial\langle H\rangle}{\partial J_{x}}=\frac{1}{2 \pi} \oint \mathrm{d} s \sum_{j, k=0}^{\infty} h_{j k} \frac{\partial\left\langle x^{2 j}\right\rangle}{\partial J_{x}}\left\langle y^{2 k}\right\rangle
$$

In the following we assume that the motion is out of any resonance. For free oscillations, $x(s)=\sqrt{2 J_{x} \beta_{x}} \cos \phi$, we have 


$$
\left\langle x^{2 j}\right\rangle=2^{-j}\left(\begin{array}{c}
2 j \\
j
\end{array}\right) \beta_{x}^{j} J_{x}^{j}
$$

giving the following amplitude detuning,

$$
\Delta Q_{x}=\frac{1}{2 \pi} \oint \mathrm{d} s \sum_{j, k=0}^{\infty} h_{j k} 2^{-j} j\left(\begin{array}{c}
2 j \\
j
\end{array}\right) \beta_{x}^{j} J_{x}^{j-1}\left\langle y^{2 k}\right\rangle .
$$

In the presence of forced oscillations the linear eigenmodes are a superposition of the two free and forced betatron oscillations [3,5,7],

$$
x(s)=\sqrt{2 J_{x} \beta_{x}} \cos \phi+\sqrt{2 A_{x} \beta_{x}^{\prime}} \cos \phi^{\prime},
$$

where $A_{x}$ is the forced action determined by the strength of the AC dipole and the distance between the natural and the driving tunes. $\beta^{\prime}$ and $\phi^{\prime}$ are the forced betatron and phase advance functions [7], which differ from the free $\beta$ and $\phi$. The $\left\langle x^{2 j}\right\rangle$ term with forced oscillations is expanded as follows,

$$
\begin{aligned}
\left\langle x^{2 j}\right\rangle & =2^{-j}\left(\begin{array}{c}
2 j \\
j
\end{array}\right) \beta_{x}^{j} A_{x}^{j}+2^{-j}\left(\begin{array}{c}
2 j \\
2 j-2
\end{array}\right)\left(\begin{array}{c}
2 \\
1
\end{array}\right)\left(\begin{array}{c}
2 j-2 \\
j-1
\end{array}\right) \beta_{x}^{j} J_{x} A_{x}^{j-1}+\mathcal{O}\left(J_{x}^{2}\right) \\
& =2^{-j}\left(\begin{array}{c}
2 j \\
j
\end{array}\right) \beta_{x}^{j} A_{x}^{j}+2^{-j} j^{2}\left(\begin{array}{c}
2 j \\
j
\end{array}\right) \beta_{x}^{j} J_{x} A_{x}^{j-1}+\mathcal{O}\left(J_{x}^{2}\right),
\end{aligned}
$$

where, as in [12], we have approximated $\beta^{\prime}=\beta$ for simplicity, which is a good approximation for LHC typical configurations. The horizontal amplitude detuning with forced oscillations is given by

$$
\Delta Q_{x}=\frac{1}{2 \pi} \oint \mathrm{d} s \sum_{j, k=0}^{\infty} h_{j k} 2^{-j} j^{2}\left(\begin{array}{c}
2 j \\
j
\end{array}\right) \beta_{x}^{j} A_{x}^{j-1}\left\langle y^{2 k}\right\rangle+\mathcal{O}\left(J_{x}\right)
$$

Note that the remaining terms represented by $\mathcal{O}\left(J_{x}\right)$ vanish for $J_{x}=0$ and that this equation holds both for free and forced vertical motion. The coefficients of the monomials of order $j-1$ in Eqs. (4) and (6) differ simply by a factor $j$. This factor can be conveniently introduced in Eq. (4) by multiplying by $J_{x}$ and taking the derivative. Therefore the forced amplitude detuning can be derived from the free detuning as

$$
Q_{x}\left(J_{y}, A_{x}\right)=\left.\frac{\partial}{\partial J_{x}}\left[J_{x} Q_{x}\left(J_{x}, J_{y}\right)\right]\right|_{J_{x}=A_{x}},
$$

where we have assumed free vertical motion, while in the case of vertical forced oscillations we get the following,

$$
Q_{x}\left(A_{x}, A_{y}\right)=\left.\frac{\partial}{\partial J_{x}}\left[J_{x} Q_{x}\left(J_{x}, J_{y}\right)\right]\right|_{J_{x}=A_{x}, J_{y}=A_{y}} .
$$

In the above equations we have used a simplified notation where the missing variables in the function $Q_{x}$ are defined to be zero, i.e. $Q_{x}\left(J_{y}, A_{x}\right) \equiv Q_{x}\left(0, J_{y}, A_{x}, 0\right)$ and $Q_{x}\left(A_{x}, A_{y}\right) \equiv Q_{x}\left(0,0, A_{x}, A_{y}\right)$. The equivalent equations for the vertical detuning are derived simply by exchanging $x$ and $y$ obtaining the following general equations,

$$
\begin{gathered}
Q_{x, y}\left(A_{x}, A_{y}\right)=\left.\frac{\partial}{\partial J_{x, y}}\left[J_{x, y} Q_{x, y}\left(J_{x}, J_{y}\right)\right]\right|_{J_{x}=A_{x}, J_{y}=A_{y}} \\
Q_{x, y}\left(J_{y, x}, A_{x, y}\right)=\left.\frac{\partial}{\partial J_{x, y}}\left[J_{x, y} Q_{x, y}\left(J_{x}, J_{y}\right)\right]\right|_{J_{x, y}=A_{x, y}} .
\end{gathered}
$$

A particularly interesting case of the equations above is the pure cross detuning, which features identical functions for free and forced oscillations,

$$
Q_{x, y}\left(A_{y . x}\right)=\left.Q_{x, y}\left(J_{y, x}\right)\right|_{J_{y, x}=A_{y, x}} .
$$

The results above are limited to only two actions larger than zero in different planes, i.e. missing $Q_{x, y}\left(J_{x}, A_{x}\right)$, $Q_{x, y}\left(J_{y}, A_{y}\right)$ and all cases with more than two actions. However, it is possible to relate free and forced polynomial terms. As an illustration we consider the one dimensional case. Let $Q_{x}\left(J_{x}\right)$ and $Q_{x}\left(J_{x}, A_{x}\right)$ have the following polynomial expansions,

$$
\begin{gathered}
Q_{x}\left(J_{x}\right)=\sum_{j=0}^{\infty} \mu_{j} J_{x}^{j}, \\
Q_{x}\left(J_{x}, A_{x}\right)=\sum_{j, k=0}^{\infty} \mu_{j k}^{\prime} J_{x}^{j} A_{x}^{k},
\end{gathered}
$$

then the $\mu_{j}$ and $\mu_{j k}^{\prime}$ coefficients are related by the following formula,

$$
\frac{\mu_{j k}^{\prime}}{\mu_{k+j}}=\frac{(j+k+1) !(j+k) !}{(j+1) ! j ! k !^{2}} .
$$

For $j=0$ we recover the factor that appeared between Eqs. (6) and (4). In the following we focus on applying the 
closed analytical formulas in Eqs. (9)-(11) to the amplitude detuning from beam-beam.

\section{BEAM-BEAM}

Assuming a round beam at the interaction point the beam-beam amplitude detuning versus the free actions is given by the following integral up to first order in the beam-beam parameter $\xi[28,29]$,

$$
\begin{aligned}
Q_{x}\left(J_{x}, J_{y}\right)= & \xi \int_{0}^{\infty} \frac{\mathrm{d} t}{(1+t)^{2}} e^{-\frac{J_{x}+J_{y}}{2 \epsilon(1+t)}} I_{0}\left(\frac{J_{y}}{2 \epsilon(1+t)}\right) \\
& \times\left[I_{0}\left(\frac{J_{x}}{2 \epsilon(1+t)}\right)-I_{1}\left(\frac{J_{x}}{2 \epsilon(1+t)}\right)\right],
\end{aligned}
$$

where $\xi$ is equivalent to the tune shift for a zero amplitude particle and for the round beam case $\xi_{x}=\xi_{y}=\xi, \epsilon$ is the beam emittance and $I_{n}(x)$ is the modified Bessel function of the first kind. The vertical detuning is obtained by swapping $x$ and $y$. This integral can only be solved when one action is zero, obtaining (for $J_{y}=0$ )

$$
\begin{gathered}
Q_{x}\left(J_{x}\right)=\xi \frac{2 \epsilon}{J_{x}}\left[1-I_{0}\left(\frac{J_{x}}{2 \epsilon}\right) e^{-\frac{J_{x}}{2 \epsilon}}\right], \\
Q_{y}\left(J_{x}\right)=\xi\left[I_{0}\left(\frac{J_{x}}{2 \epsilon}\right)+I_{1}\left(\frac{J_{x}}{2 \epsilon}\right)\right] e^{-\frac{J_{x}}{2 \epsilon}} .
\end{gathered}
$$

Applying the transformation given in Eqs. (9) and (11) to Eqs. (15) and (16) and using that

$$
\frac{d I_{0}(x)}{d x}=I_{1}(x)
$$

the approximations to the horizontal and vertical beambeam detuning of forced oscillations are given by

$$
\begin{aligned}
& Q_{x}\left(A_{x}\right)=\xi\left[I_{0}\left(\frac{A_{x}}{2 \epsilon}\right)-I_{1}\left(\frac{A_{x}}{2 \epsilon}\right)\right] e^{-\frac{A_{x}}{2 \epsilon}} . \\
& Q_{y}\left(A_{x}\right)=\xi\left[I_{0}\left(\frac{A_{x}}{2 \epsilon}\right)+I_{1}\left(\frac{A_{x}}{2 \epsilon}\right)\right] e^{-\frac{A_{x}}{2 \epsilon}},
\end{aligned}
$$

Figure 1 shows 2-dimensional footprints from free and forced oscillations numerically computed from Eq. (14) and its transformation according to Eq. (9), respectively. Figure 2 shows the relative beam-beam amplitude detuning for free and forced oscillations in one dimension from the analytical formulas in Eqs. (15) and (18). Both curves have same initial and asymptotic values as expected intuitively. At small amplitudes, forced oscillations detune a factor two faster than free oscillations as expected. Forced oscillations approach the asymptote considerably faster.

Single particle tracking simulations have been performed with MAD-X [30] to verify Eqs. (18) and (19). Figure 3
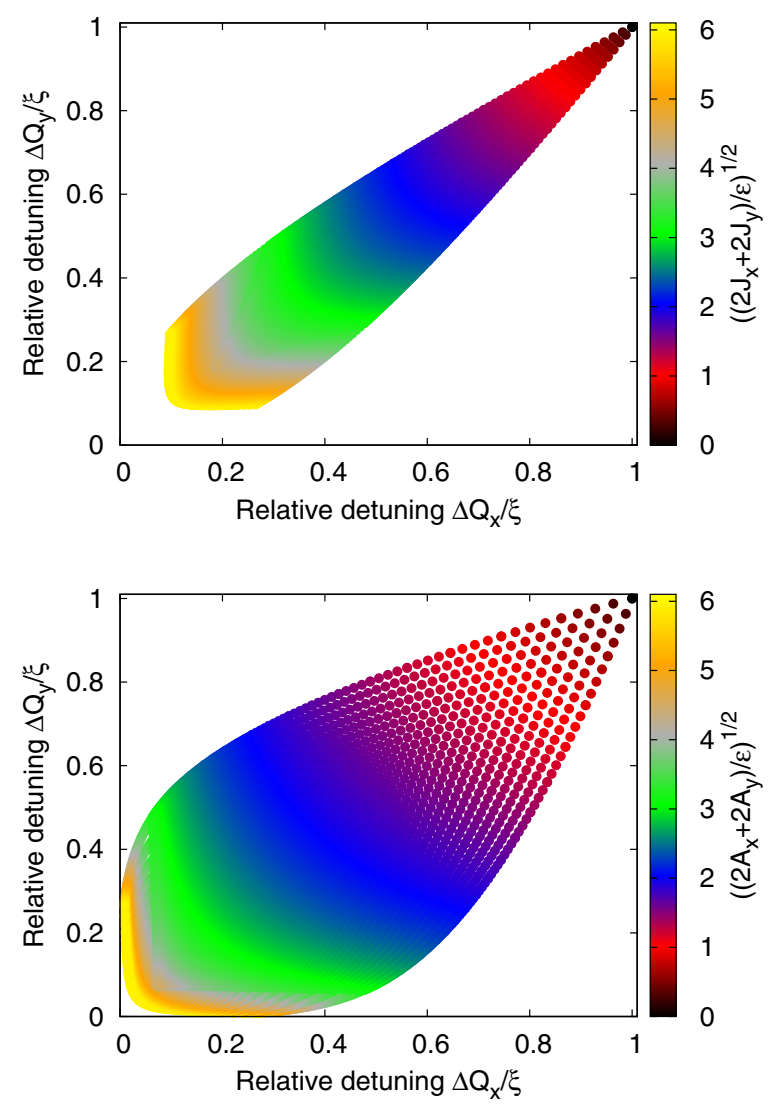

FIG. 1. Beam-beam footprint from free (top) and forced (bottom) oscillations from Eq. (14) and its transformation according to Eq. (9), respectively. The radial oscillation amplitude is represented by the color map in units of beam sigma.

shows the tune shift versus amplitude for the LHC, with one head-on encounter, $\xi=0.0074$, bare machine fractional tunes of $Q_{x}=0.31, Q_{y}=0.32$ and driving horizontal tune of 0.292 . The forced oscillation is obtained by ramping up an $\mathrm{AC}$ dipole in 4000 turns.

The equations presented above should also be relevant when two beams interact in the weak-strong regime and the

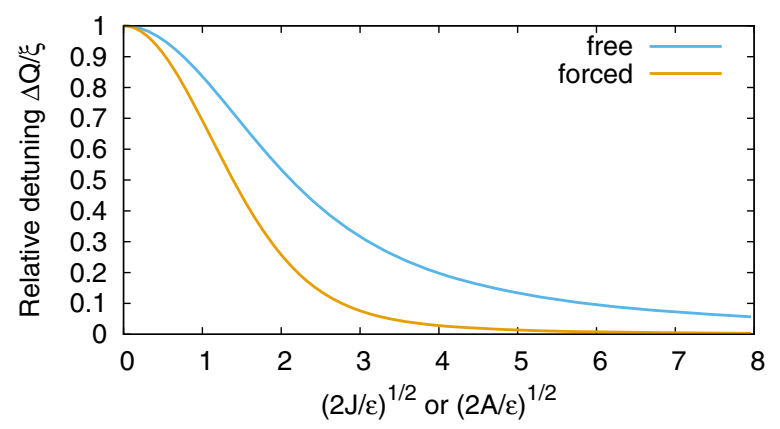

FIG. 2. Beam-beam amplitude detuning for free and forced oscillations in one dimension, Eqs. (15) and (18). The horizontal axis represents the oscillation amplitude normalized to the beam size. 

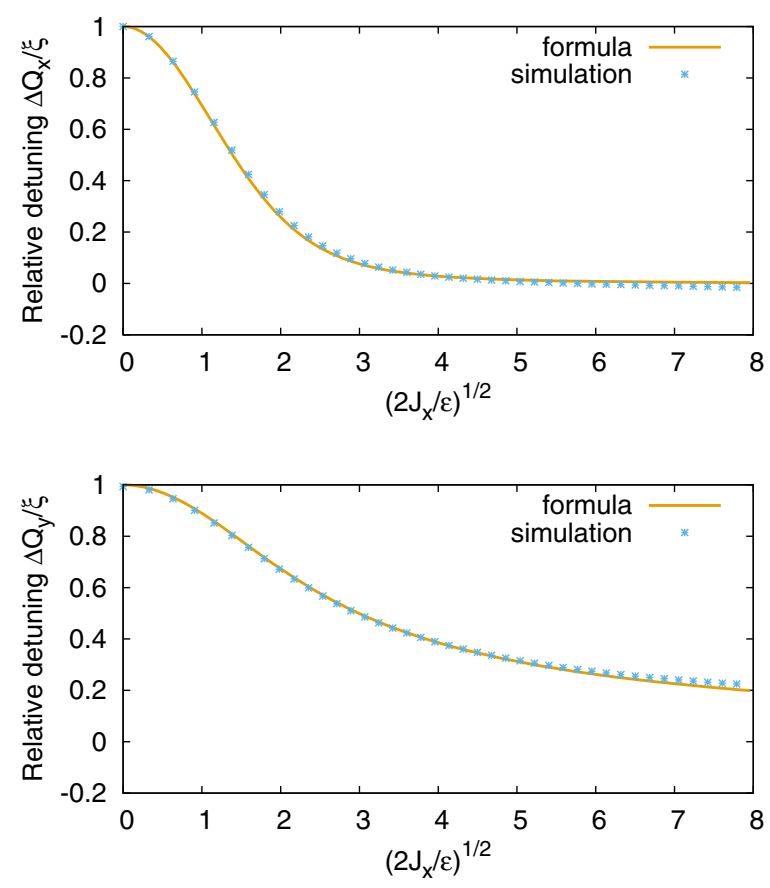

FIG. 3. Comparison of forced beam-beam amplitude detuning analytical formulas to simulations. Top: Direct amplitude detuning from Eq. (18). Bottom: Cross amplitude detuning from Eq. (19).

forced oscillation is applied to the weak beam. The limitation is that the weak beam features a distribution in the free actions $J_{x}$ and $J_{y}$ which are not present in the closed analytical equations in Eqs. (18) and (19). We illustrate the behavior of $Q\left(J_{x}, A_{x}\right)$ via single particle simulations. The free action corresponds to an initial offset of the particle. The forced action is computed from the Fourier amplitude of the spectral line with the forced tune during the excitation plateau. Particles with larger free action $\left(J_{x}\right)$ feature a weaker detuning versus the forced action $\left(A_{x}\right)$ as shown in Fig. 4 with simulations. The

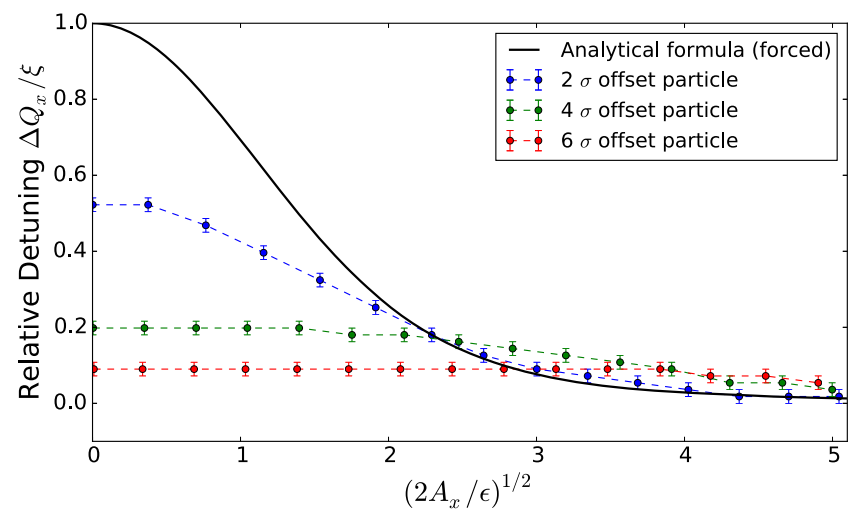

FIG. 4. Simulated relative amplitude detuning versus forced oscillation amplitude for particles with increasing free oscillation amplitudes: $2 \sigma, 4 \sigma$ and $6 \sigma$. Equation (18) is also shown representing the $0 \sigma$ particle. detuning for particles at larger free amplitudes cross the analytical formula $\left(J_{x}=0\right)$ between $2 \sigma$ and $3 \sigma$. Therefore the analytical formula should represent the upper boundary of the incoherent spectrum of the weak beam motion for forced oscillations below $2 \sigma$ and the lower boundary for forced oscillations above $3 \sigma$. Such an effect is not present in the nonexcited plane and the analytical formula should represent the upper boundary of the incoherent spectrum. This can be understood from the shape of the beam-beam force since its derivative changes sign at about $1.6 \sigma$. A similar behavior is observed when beams collide with a fixed offset [31,32].

A similar behavior is observed for the detuning in collisions with fixed beams separation [31].

LHC multiparticle simulations have also been performed with COMBI [33] in the weak-strong regime to compare to the single particle analytical formula. Beam distributions are considered with equal emittances of $2 \mu \mathrm{m}$ in the transverse planes for both beams. The intensity ratio is 100 between the strong and the weak beams and a transverse feedback is activated on the strong beam with a damping time of 20 turns. The beam-beam parameter is $\xi=0.0061$ with one beam-beam interaction. The bare machine fractional tunes are $Q_{x}=0.31, Q_{y}=0.32$ and

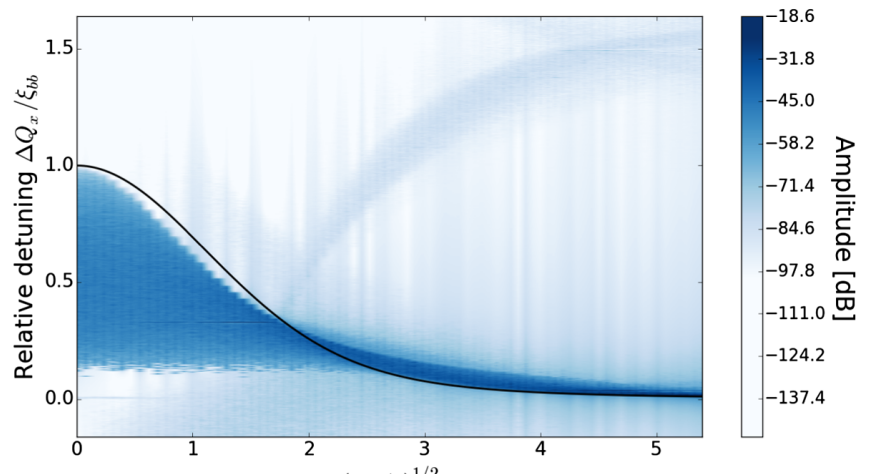

$(2 A / \epsilon)^{1 / 2}$

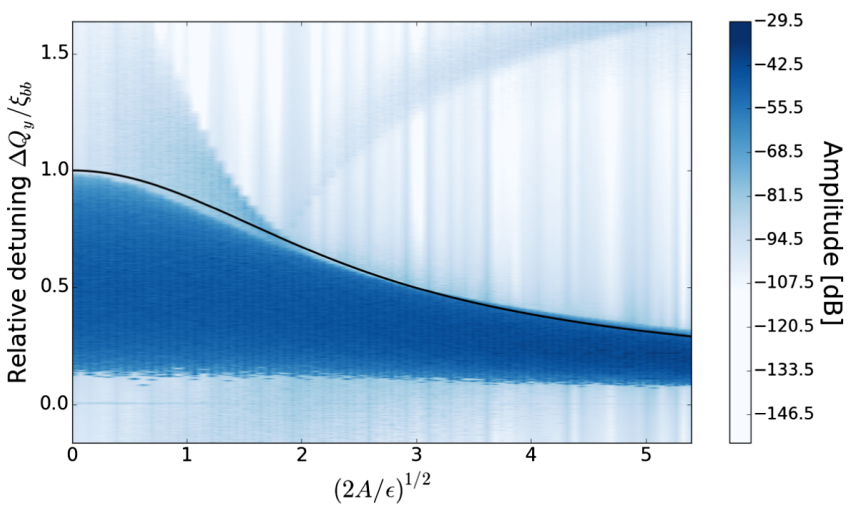

FIG. 5. Simulated frequency spectrograms for the horizontal (top) and vertical (bottom) planes versus horizontal amplitude of forced oscillations in the weakly interacting beam. The black curves correspond to the single particle forced beam-beam amplitude detuning analytical formulas in Eqs. (18) and Eq. (19). 
the driving horizontal tune is 0.292 . The forced oscillation is obtained by ramping the AC dipole in 2000 turns and the beams are tracked for $10^{6}$ turns with $10^{5}$ macroparticles. The Fourier transform is computed to the horizontal and vertical turn-by-turn centroid positions for a fine scan of forced oscillation amplitudes. Figure 5 shows these Fourier spectra in color code versus forced oscillation amplitude together with the analytical formula. These multiparticle simulations confirm the usefulness of the analytical formulas in the weak-strong regime. In the nonexcited plane analytical formula represents the upper boundary of the incoherent spectrum, Fig. 5 bottom. In the excitation plane, Fig. 5 top, the analytical formula changes between the upper and lower boundaries in the range of $2 \sigma$ and $3 \sigma$ forced amplitude, as expected from the above single particle simulations.

\section{CONCLUSIONS}

Generalized analytical relations between free and forced amplitude detuning functions have been derived. These have been applied to the case of the beam-beam interaction being the source of amplitude detuning and simulations with the LHC model have been used to verify the analytical formula. The applicability of the formulas in the weakstrong regime has been demonstrated with multiparticle simulations.

\section{ACKNOWLEDGMENTS}

Thanks to Massimo Giovannozzi for proofreading the manuscript. We are very thankful to F. Carlier and W. Fischer for fruitful and motivating discussions.

[1] K. Ohmi, R. Tomás, Y. Funakoshi, R. Calaga, T. Ieiri, Y. Morita, K. Nakanishi, K. Oide, Y. Ohnishi, Y. Sun, M. Tobiyama, and F. Zimmermann, Response of colliding beam-beam system to harmonic excitation due to crabcavity rf phase modulation, Phys. Rev. ST Accel. Beams 14, 111003 (2011).

[2] M. Bai et al., Overcoming Intrinsic Spin Resonances with an rf Dipole, Phys. Rev. Lett. 80, 4673 (1998).

[3] S. Peggs and C. Tang, Report No. RHIC/AP/159, 1998, http://www.agsrhichome.bnl.gov/AP/ap_notes/RHIC_AP_ 159.pdf.

[4] M. Bai, J. Delong, L. Hoff, C. Pai, S. Peggs, J. Piacentino, B. Oerter, P.Oddo, T. Roser, T. Satogata, D. Trbojevic, and A. Zaltsman, in Proceedings of the 8th European Particle Accelerator Conference, Paris, 2002 (EPS-IGA and CERN, Geneva, 2002), p. 115, http://accelconf.web.cern .ch/AccelConf/e02/PAPERS/WEPLE040.pdf.

[5] R. Tomás, Normal form of particle motion under the influence of an AC dipole, Phys. Rev. ST Accel. Beams 5, 054001 (2002).

[6] R. Tomás, Adiabaticity of the ramping process of an ac dipole, Phys. Rev. ST Accel. Beams 8, 024401 (2005).
[7] R. Miyamoto, S. E. Kopp, A. Jansson, and M. J. Syphers, Parametrization of the driven betatron oscillation, Phys. Rev. ST Accel. Beams 11, 084002 (2008).

[8] R. Tomás, O. Brüning, M. Giovannozzi, P. Hagen, M. Lamont, F. Schmidt, G. Vanbavinckhove, M. Aiba, R. Calaga, and R. Miyamoto, CERN Large Hadron Collider optics model, measurements, and corrections, Phys. Rev. ST Accel. Beams 13, 121004 (2010).

[9] R. Tomás, T. Bach, R. Calaga, A. Langner, Y. I. Levinsen, E. H. Maclean, T. H. B. Persson, P. K. Skowronski, M. Strzelczyk, G. Vanbavinckhove, and R. Miyamoto, Record low beta-beating in the LHC, Phys. Rev. ST Accel. Beams 15, 091001 (2012).

[10] T. Persson and R. Tomás, Improved control of the betatron coupling in the Large Hadron Collider, Phys. Rev. ST Accel. Beams 17, 051004 (2014).

[11] T. Persson, F. Carlier, J. Coello de Portugal, A. GarciaTabares Valdivieso, A. Langner, E. H. Maclean, L. Malina, P. Skowronski, B. Salvant, R. Tomás, and A. C. Garcia Bonilla, LHC optics commissioning: A journey to the $1 \%$ optics control, Phys. Rev. Accel. Beams 20, 061002 (2017).

[12] S. White, E. Maclean, and R. Tomás, Direct amplitude detuning measurement with ac dipole, Phys. Rev. ST Accel. Beams, 16, 071002 (2013).

[13] X. Shen, S. Y. Lee, M. Bai, S. White, G. RobertDemolaize, Y. Luo, A. Marusic, and R. Tomás, Application of independent component analysis to ac dipole based optics measurement and correction at the Relativistic Heavy Ion Collider, Phys. Rev. ST Accel. Beams 16, 111001 (2013).

[14] S. Mönig, E. H. Maclean, T. H. B. Persson, J. Coello de Portugal, A. Langner, and R. Tomás, in Proceedings of 7th InternationalParticle Accelerator Conference, Busan, Korea, edited by C. Petit-Jean-Genaz, D. E. Kim, K. S. Kim, I. S. Ko, K. R. Kim, and V. RW Schaa (2016), p. 3496; Report No. CERN-ACC-NOTE-2015-0027, 2015; http://accelconf .web.cern.ch/AccelConf/ipac2016/papers/thpmr044.pdf.

[15] R. Tomás, M. Aiba, A. Franchi, and U. Iriso, Review of linear optics measurement and correction for charged particle accelerators, Phys. Rev. Accel. Beams 20, 054801 (2017).

[16] P. Görgen, O. Boine-Frankenheim, and W. Fischer, Beam transfer functions for relativistic proton bunches with beam-beam interaction, Nucl. Instrum. Methods Phys. Res., Sect. A 777, 43 (2015).

[17] C. Tambasco, T. Pieloni, X. Buffat, A. Boccardi, K. Fuchsberger, M. Gasior, R. Giachino T. Lefevre, T. Levens, M. Pojer, B. Salvachua, M. Solfaroli, and J. Barranco, in Proceedings of IPAC 2016, Busan, Korea (JACoW, Busan, 2016), p. 3051.

[18] C. Tambasco, PhD thesis n. 7867, EPFL Lausanne, 2017.

[19] N. Biancacci and R. Tomás, Using AC dipoles to localize sources of beam coupling impedance, Phys. Rev. Accel. Beams 19, 054001 (2016).

[20] T. Pieloni, X. Buffat, L. E. Medina Medrano, C. Tambasco, R. Tomás, J. Barranco, P. Gonçalves Jorge, and C. Tambasco, in HB2016, Malmö, Sweden (JACoW, Malmö, 2016), https://hb2016.esss.se/prepress/papers/mopr027.pdf.

[21] P. Gonçalves Jorge, J. Barranco, T. Pieloni, X. Buffat, F. S. Carlier, J. Coello de Portugal, E. Fol, L. Medina, R. Tomás, and A. Wegscheider, IPAC 2017, Copenhagen, Denmark (JACoW, Copenhagen, 2017), p. 2121. 
[22] P. Gonçalves Jorge, Master thesis, EPFL Lausanne, 2017.

[23] X. Gu et al., Electron lenses for head-on beam-beam compensation in RHIC, Phys. Rev. Accel. Beams 20, 023501 (2017).

[24] Simulations and measurements on long-range beam-beam effects in the LHC workshop, Lyon, France, November 2015, https://indico.cern.ch/event/456856.

[25] Second Workshop on Wire Experiment for Long Range Beam-Beam Compensation, Divonne, France, March 2017, https://indico.cern.ch/event/615088/.

[26] J. Barranco, T. Pieloni, X. Buffat, and C. Tambasco, Proceedings of IPAC2017, Copenhagen, Denmark (JACoW, Copenhagen, 2017), p. 2113.

[27] L. Medina, R. Tomás, X. Buffat, J. Barranco, and T. Pieloni, Proceedings of IPAC 2017, Copenhagen, Denmark (JACoW, Copenhagen, 2017), p. 2512.
[28] A. W. Chao, Report No. SLAC-PUB-3179, 1983, http:// www.iaea.org/inis/collection/NCLCollectionStore/_Public/ 15/021/15021887.pdf.

[29] A. W. Chao, P. Bambade, and W. T. Weng, Report No. SLAC-PUB-3545, 1985, http://www.slac.stanford .edu/cgi-wrap/getdoc/slac-pub-3545.pdf.

[30] http://madx.web.cern.ch/madx/.

[31] M. Meddahi and R. Schmidt, Report No. CERN-SL-90-15AP, 1990.

[32] G. López, in Proceedings of the 15th Particle Accelerator Conference, PAC-1993, Washington, DC, 1993 (IEEE, New York, 1993), p. 3467, https://lib-extopc.kek.jp/ preprints/PDF/1991/9109/9109217.pdf.

[33] T. Pieloni, thesis École polytechnique fédérale de Lausanne, 2008. 